\title{
Uso de Aeronaves Remotamente Pilotadas (RPA) en la estimación de poblaciones de lobos marinos en la Isla Chincha sur - Ica
}

Recibido: 20/10/2019 Aceptado: 24/06/2020 Publicado: 31/12/2020

\author{
FABIÁN BRONDI RUEDA \\ REgina E. AgUilar ARAKAKI ${ }^{2}$ \\ JHON CANDELARIO FANO ${ }^{3}$
}

\begin{abstract}
RESUMEN
Esta publicación describe el empleo de la tecnología RPA como método complementario durante el censo de lobos marinos chuscos (Otaria flavescens), en la Isla Chincha sur, Ica - Perú, como parte de las actividades programadas por el Instituto del Mar del Perú (IMARPE). Se llevó a cabo el mapeo de las colonias de estos pinnípedos y su posterior clasificación en entorno de Sistemas de Información Geográfico, con el objetivo de comparar los resultados obtenidos por conteo directo en el campo y el conteo de ortofotos generadas por RPA. Los valores totales de los conteos del método directo y de las ortofotos fueron 2300 y 2700 individuos, respectivamente. Si bien no se encontraron diferencias significativas entre ambos conteos, el conteo digital permitió obtener un mayor detalle del número de individuos correspondientes a las categorías crías y hembras. El uso de RPA constituye una herramienta que permite la georreferenciación de cada individuo y la posibilidad de hacer repeticiones del conteo, incluso por diferentes evaluadores.
\end{abstract}

Palabras clave: Otaria flavescens; Aeronaves Remotamente Pilotadas; RPA; CENICAL; fotogrametría; sistemas de información geográfico.

Use of Remotely Piloted Aircraft (RPA) in THE ESTIMATION OF SEA LION POPULATIONS IN SOUTH CHINCha ISLAND - ICA

\section{ABSTRACT}

This paper describes the use of RPA technology as a complementary method during Southern sea lions (Otaria flavescens) counting in Chincha Sur Island, Ica - Peru, as part of the activities scheduled by the Instituto del Mar del Perú (IMARPE). Colonies of these pinnipeds were mapped and its subsequent classification in the Systems of Geographic Information was carried out in order to make comparisons between direct counting on the field and RPA mapping counts. Total values by direct counting and orthophotos were 2300 and 2700 individuals, respectively. While no significant differences were found between the two methods, by means of digital counting, a better detail of the number of individuals on the females and pups categories could be achieved. The use of RPA constitutes a valuable tool that provides geographical reference of each individual and enables repetitions of the counts by different people.

Keywords: Otaria flavescens; remotely piloted aircraft; RPA; CENICAL; fotogrametry; geographic information systems.

\section{INTRODUCCIÓN}

Durante la evaluación realizada en Isla Chincha Sur, el IMARPE usó por primera vez la tecnología de RPA, apoyado por el Instituto Geográfico Nacional para la obtención de registros fotogramétricos de la población de lobos marinos, los cuales constituyen herramientas confiables para la determinación de abundancia y densidad de colonias de pinnípedos, básicamente se generó un mapa con referencia espacial de cada individuo censado. Esta metodología puede ser aplicada posteriormente a otras especies, y/o llevar a cabo estudios relacionados a los hábitats ocupados, comportamiento o condición de los animales, entre otros (Johnston, 2019). Adicionalmente, representa ventajas en el campo para los operadores y los animales evaluados, ya que cuando son usados de manera cuidadosa, son seguros para los investigadores y no ponen en riesgo a los animales ya que no es invasiva; además de cubrir amplias extensiones de terreno (Sweeney, Helker, Perryman, LeRoi, Fritz, Gelatt y Angliss, 2016) en una sola salida de campo, y la posibilidad de realizar conteos de fotografías en gabinete con mayor precisión, minimizando los errores de conteo directo (Hodgson, Mott, Baylis, Pham, Wotherspoon, Kilpatrick, Raja Segaran, Reid, Terauds. y Koh, 2018).

El monitoreo de fauna silvestre constituye una herramienta importante para determinar estados y tendencias de las poblaciones, así como la asociación a diferentes causas que pudieran afectarlas (Mandujano, Mulero-Pázmany y Rísquez-Valdepeña, 2017) y que están relacionadas con cambios en el ambiente o factores antropogénicos, actividad importante para los ecosistemas marinos.

Los censos de pinnípedos se realizan mediante el conteo directo de individuos en las playas de agregación y reproducción, ya

1 Ms. C., Director del Departamento de Cartografía Especial del Instituto Geográfico Nacional del Perú. Lima - Perú

ORCID: https://orcid.org/0000-0002-1582-4892

E-mail: nbrondir@ign.gob.pe

2 Bachiller, Oficina de Investigaciones en Depredadores Superiores - Instituto del Mar del Perú. Lima - Perú

ORCID: https://orcid.org/0000-0002-1075-600X

E-mail: raguilar@imarpe.gob.pe

3 Licenciado, Sub director del Departamento de Cartografía Especial del Instituto Geográfico Nacional del Perú. Lima - Perú

ORCID: https://orcid.org/0000-0002-2985-6350.

E-mail: jcandelariof@ign.gob.pe 
sea desde tierra o desde embarcaciones en el mar; o fotografiando la población total o en segmentos para después contar los individuos en las fotografías (Berovides, Cañizares y González, 2005). En países como Argentina y Chile, se realiza parte de la estimación de las poblaciones de lobos marinos mediante el uso de aeronaves tripuladas, especialmente cuando se trata de cubrir grandes extensiones de terreno y lugares inaccesibles por tierra o por vía marítima (Eberhardt, Chapman y Gilbert, 1979; Dans Crespo, Pedraza y Koen, 2004; Sepúlveda, Oliva, Urra, Pérez-Álvarez, Moraga, Schrader, Inostroza, Melo, Díaz y Sielfeld, 2011).

Cada método presenta ventajas; así, el conteo directo de individuos en el campo presenta ventajas comparado con el uso de aeronaves tripuladas, como obtener resultados el mismo día de la ejecución de la actividad, al no necesitar procesamiento de fotografías, ni conteo de gabinete (Arias-Schreiber y Rivas, 1998). En contraparte, supone la disponibilidad de un mayor número de personas, con experiencia en la identificación de animales de modo que los errores de conteo sean minimizados, para abarcar varias localidades y hacer los conteos de manera simultánea (Contreras, Bartheld, Montecinos, Moreno y Torres, 2014).

Por otro lado, los conteos de fotografías tomadas desde aeronaves permiten hacer un trabajo de gabinete con mayor detenimiento y precisión (Sepúlveda et al., 2011); sin embargo, supone desventajas como ruido de motores que podría impactar y espantar a los animales, mayor gasto económico que involucra el uso de aviones y/o helicópteros, detalles logísticos y operativos a tener en cuenta; además de los riesgos personales que implica el estar a bordo de un vehículo aéreo (Mandujano y Rísquez-Valdepeña, 2019; McEvoy, Hall y McDonald, 2016).

El objetivo de esta publicación es documentar la estimación de la población de lobos marinos chuscos Otaria flavescens de la isla Chincha Sur, como parte de la evaluación anual de abundancia de esta especie que realiza el Instituto del Mar del Perú, empleando la metodología de vuelo cenital fotogramétrico, mediante el uso de aeronaves pilotadas remotamente, y su posterior conteo en un entorno de sistemas de información geográfico (SIG), y comparación con el método de conteo directo tradicional.

\section{METODOLOGÍA}

\section{Área de estudio}

Este estudio se desarrolló el día 01 de marzo del 2019, en la isla Chincha Sur $\left(-13.651225^{\circ}\right.$, $-76.401639^{\circ}$ ), ubicada a $21 \mathrm{~km}$ del puerto de San Andrés, en la ciudad de Pisco, Departamento de Ica (Figura 1).

La isla alberga especies de aves marinas, como el piquero peruano (Sula variegata), pelícano peruano (Pelecanus thagus), guanay (Leucocarbo bougainvillii), zarcillo (Larosterna inca), entre otras; y una población estimada superior a los 3000 individuos en los últimos cinco años (IMARPE, datos no publicados) de lobos marinos chuscos Otaria flavescens.

\section{Mapeo fotogramétrico con RPA}

La primera tarea consistió en realizar un vuelo de reconocimiento RPA, sobre la zona de interés y alrededores, con la finalidad de establecer la altura de vuelo que permita generar un tamaño de pixel óptimo, que facilite la identificación de la especie de interés teniendo en consideración evitar la perturbación de la fauna del lugar.

Finalizado el reconocimiento, se utilizó la aplicación DJI GS Pro para elaborar el polígono y plan de vuelo, los cuales comprendieron la zona por mapear. Se aplicó la metodología de vuelo fotogramétrico, que permite tomar fotografías sucesivas con enfoque cenital (Figura 2), correlacionadas tanto en el eje " $X$ " como en el eje "Y".

Las misiones de vuelo fueron ejecutadas entre las 06:20 y 08:30, ya que ese es el horario promedio en que los animales están descansando en tierra, pudiendo encontrarlos en mayor número sobre las playas (Arias-Schreiber y Rivas, 1998; Pavanato, Silva, Estima, Monteiro y Kinas, 2013). Se realizó un solo plan de vuelo el cual con un intercambiando de cuatro baterías, este tuvo los siguientes parámetros (Tabla 1 y figura 3 )

Tabla 1. Parámetros establecidos para el mapeo de la Isla Chincha Sur.

\begin{tabular}{|l|l|}
\hline \multicolumn{1}{|c|}{ Equipo RPA } & \multicolumn{1}{c|}{ Phantom 4 Pro } \\
\hline Tiempo de vuelo & 40 minutos \\
\hline Modelo de cámara & P4 Pro 20 mgp \\
\hline Área cubierta & 60 hectáreas aprox. \\
\hline Ángulo de vuelo & 258 grados \\
\hline Líneas de vuelo & 30 \\
\hline Altura & 72 metros \\
\hline Velocidad & $7 \mathrm{~m} / \mathrm{seg}$ \\
\hline Resolución & $2 \mathrm{~cm} / \mathrm{px}$ \\
\hline Traslape horizontal & $80 \%$ \\
\hline Traslape vertical & $70 \%$ \\
\hline Cantidad de baterías empleadas & 4 \\
\hline Cantidad de fotos tomadas & 1000 \\
\hline
\end{tabular}

Fuente: Elaboración propia. 


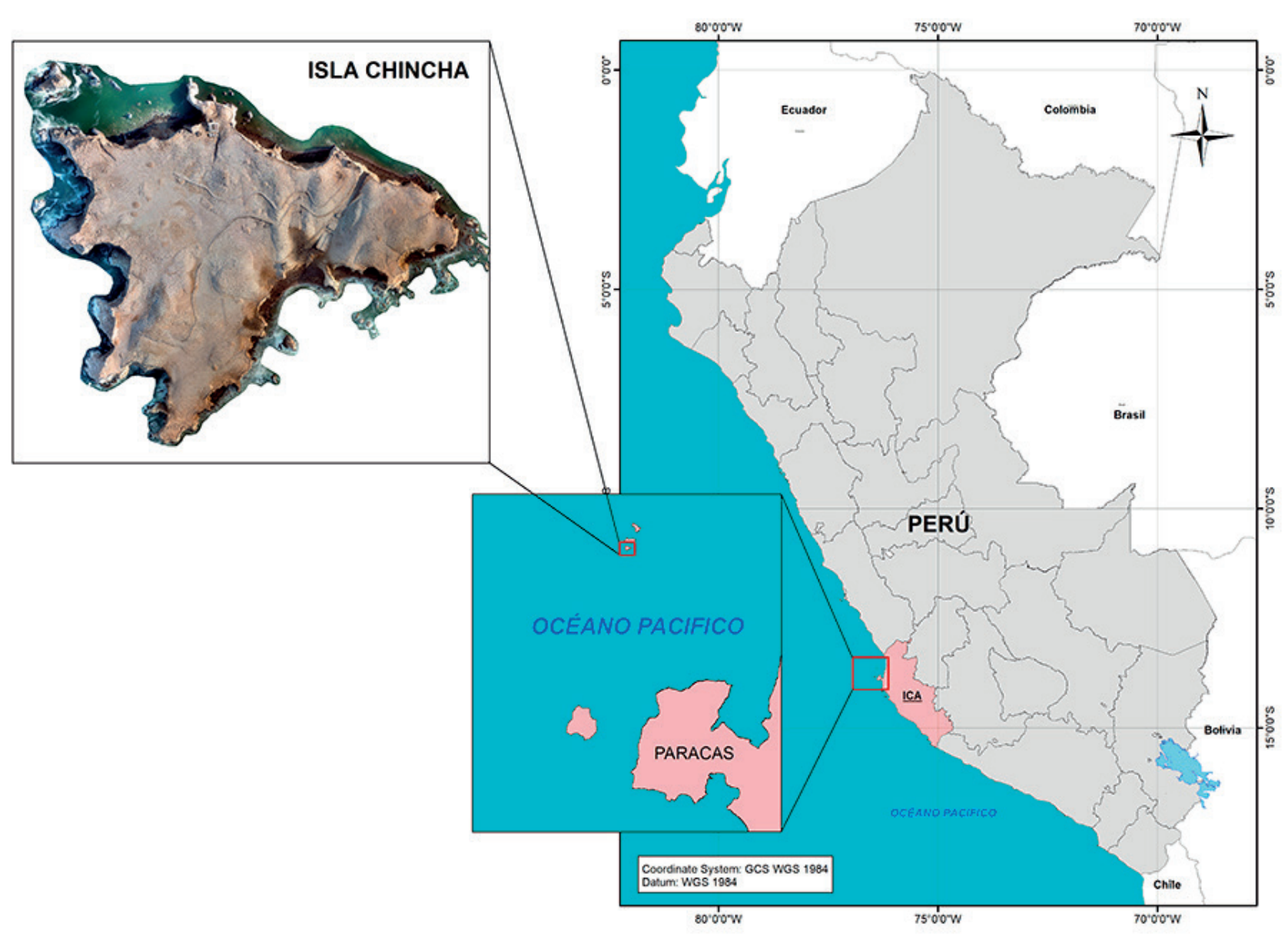

Figura 1. Ubicación de la Isla Chincha Sur, frente a la costa del Departamento de Ica. Fuente: Elaboración propia.

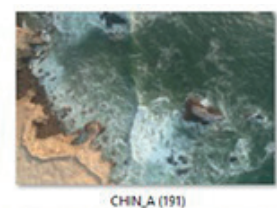

CHNA(191)

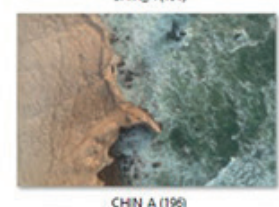

Cana $(100)$

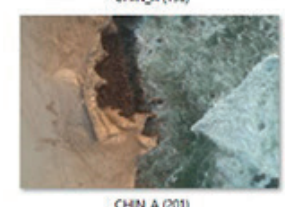

CHNA (201)

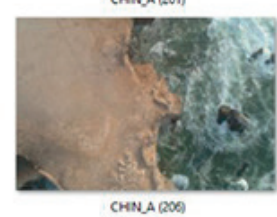

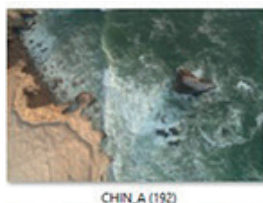

CHNA (192)

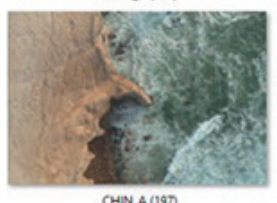

chences

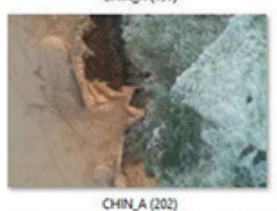

CHNA(202)

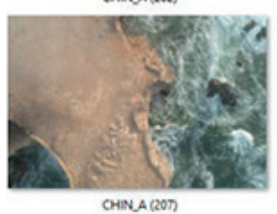

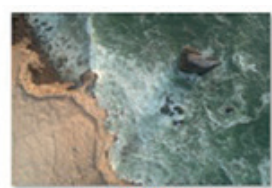

CHenA(193)

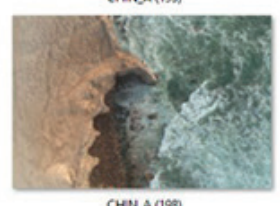

chenta

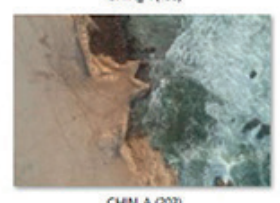

CHENACOBS

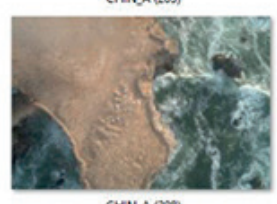

CHELACOCOO)

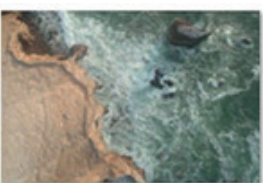

CHINA (ISA)

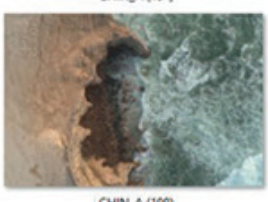

CHIN.A (195)

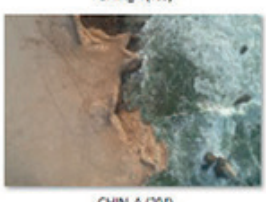

OHINAA(20)

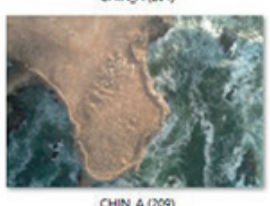

CHIN.A (200)

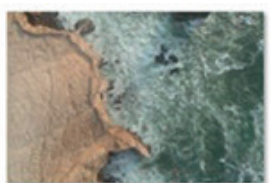

CHNLA (195)

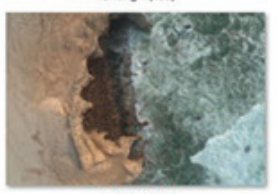

CHNAPDOS

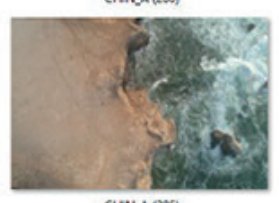

CHNACOOS)

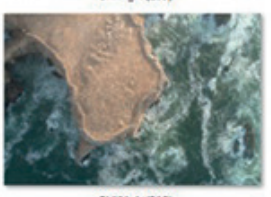

CHENAR (210)

Figura 2. Correlación de fotografías tomadas con enfoque cenital, correlacionadas tanto en el eje " $\mathrm{X}$ " como en el eje "Y".

Fuente: Elaboración propia. 


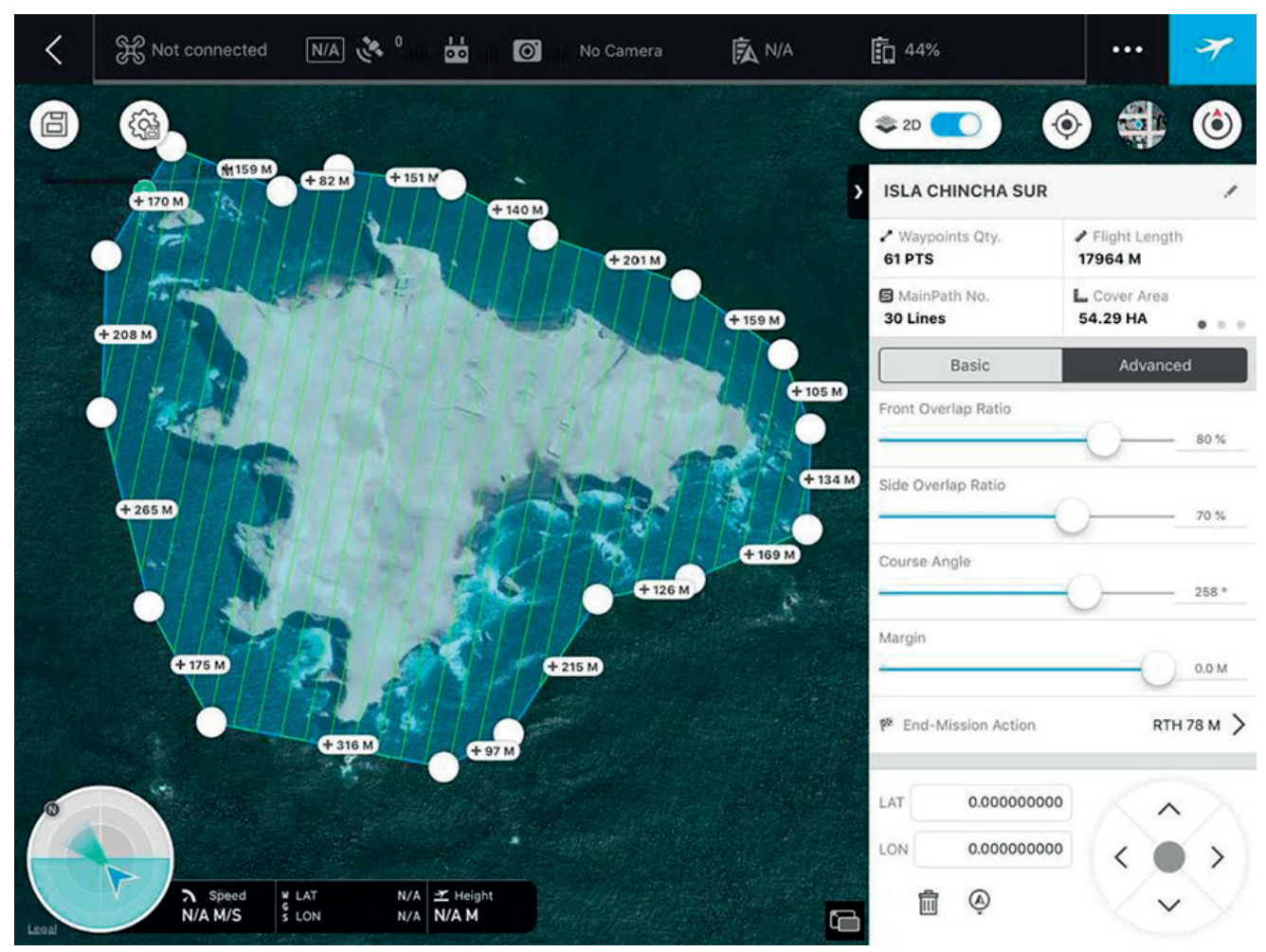

Figura 3. Captura de pantalla durante la generación de parámetros de plan de vuelo fotogramétrico, haciendo empleo de aplicación DJI GS PRO.

Fuente: Elaboración propia.

\section{Procesamiento de imágenes}

Obtenidas las imágenes digitales fueron descargadas, revisadas y procesadas en el entorno del programa Pix 4D (Figura 4), el flujo de procesamiento fue el siguiente:

1. Proceso inicial.

Se cargaron las imágenes del área de mapeo, los puntos llave fueron elegidos en alta calidad y el método de calibración elegido fue el de orientación y geolocalización de precisión.

2. Nube de puntos densa y malla.

En esta etapa se construyó la nube de puntos densa en alta calidad, con una densificación de puntos alta, el parámetro elegido para la generación de la malla y textura fue el de alta resolución con balance de color.

3. Ortomosaico y modelo en tres dimensiones.

Cumplidas las etapas anteriores, se configuró el proyecto para obtener una ortofoto con resolución de 2 centímetros por pixel y un modelo en tres dimensiones de la isla Chincha Sur.

\section{Conteo directo de lobos marinos}

Se realizó el conteo de lobos marinos chuscos, mediante observación directa entre las 6:20 y 9:000 h utilizando binoculares de aumento $10 \times 50$ y contómetro desde la parte alta de los acantilados de la isla para tener una visión directa de las playas que albergan individuos (Figura 5).

Se registró el número total de individuos de las playas (Arias-Schreiber y Rivas, 1998); los individuos censados fueron categorizados según edad y sexo en: machos adultos, machos subadultos, crías (individuos nacidos ese año), hembras adultas, juveniles, y no determinados, según sus respectivos caracteres secundarios (Vaz-Ferreira, 1982; Oliva et al., 2012) (Figura 6).

Machos adultos: Poseen un tamaño superior a todos los demás individuos de la colonia $(2.1$ - 2.6 $\mathrm{m})$. Melena larga amarillenta evidente, alrededor del cuello; hocico romo, cuello macizo. Cintura pélvica estrecha en relación a la cintura escapular.

Machos subadultos: Longitud inferior a 2 metros, cuello grueso, hocico romo y cintura pélvica estrecha. Sólo indicios de melena. Suelen encontrarse en la periferia de la colonia. 

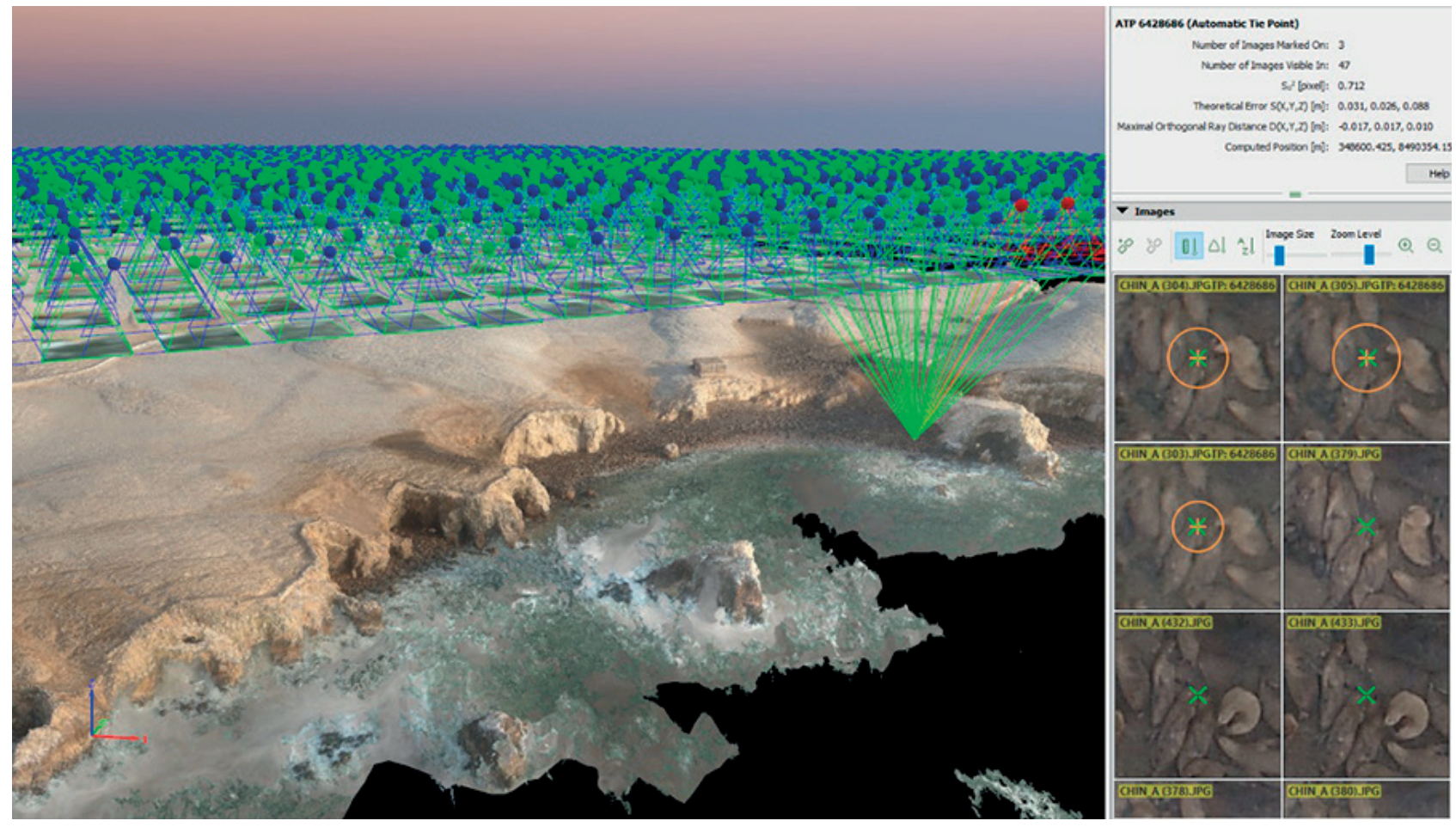

Figura 4. Captura de pantalla durante las etapas de procesamiento en entorno Pix 4d, se puede apreciar en la parte superior el alineamiento de las fotografías tomadas de manera cenital, nube puntos, y construcción del modelo en tres dimensiones de la Isla Chincha Sur.

Fuente: Elaboración propia.

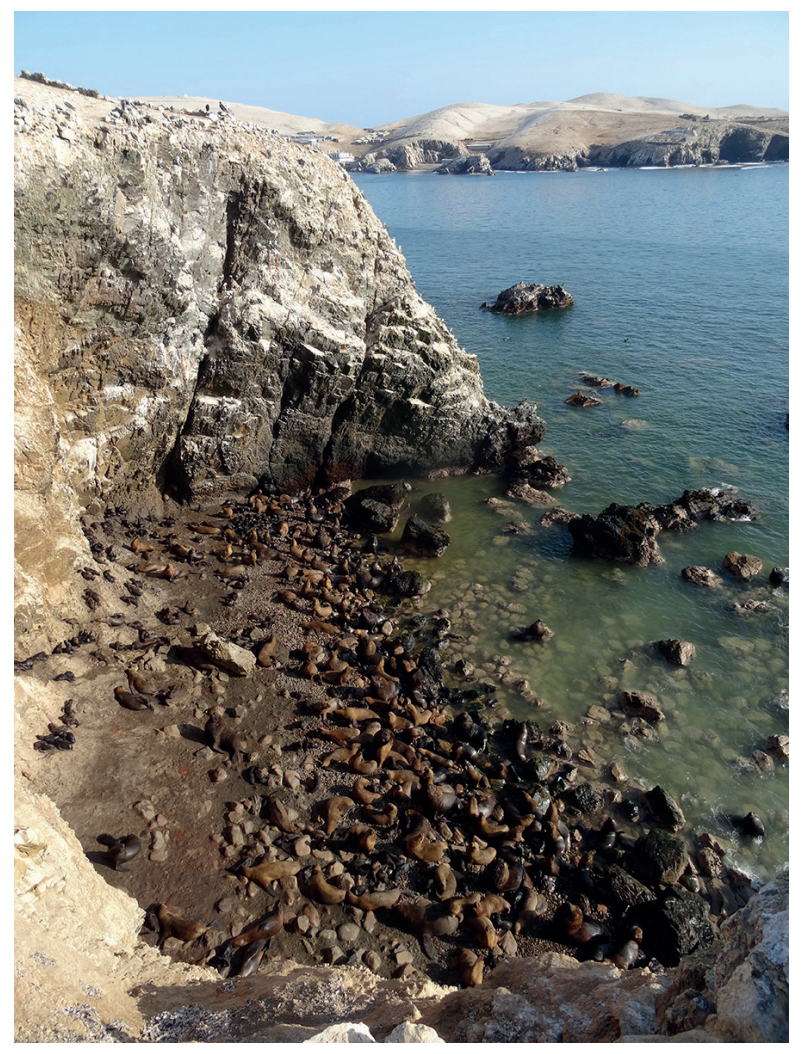

Figura 5. Vista de una playa que alberga lobos marinos desde la ubicación de conteos directos en la parte alta de superior del acantilado.

Fuente: Elaboración propia. 


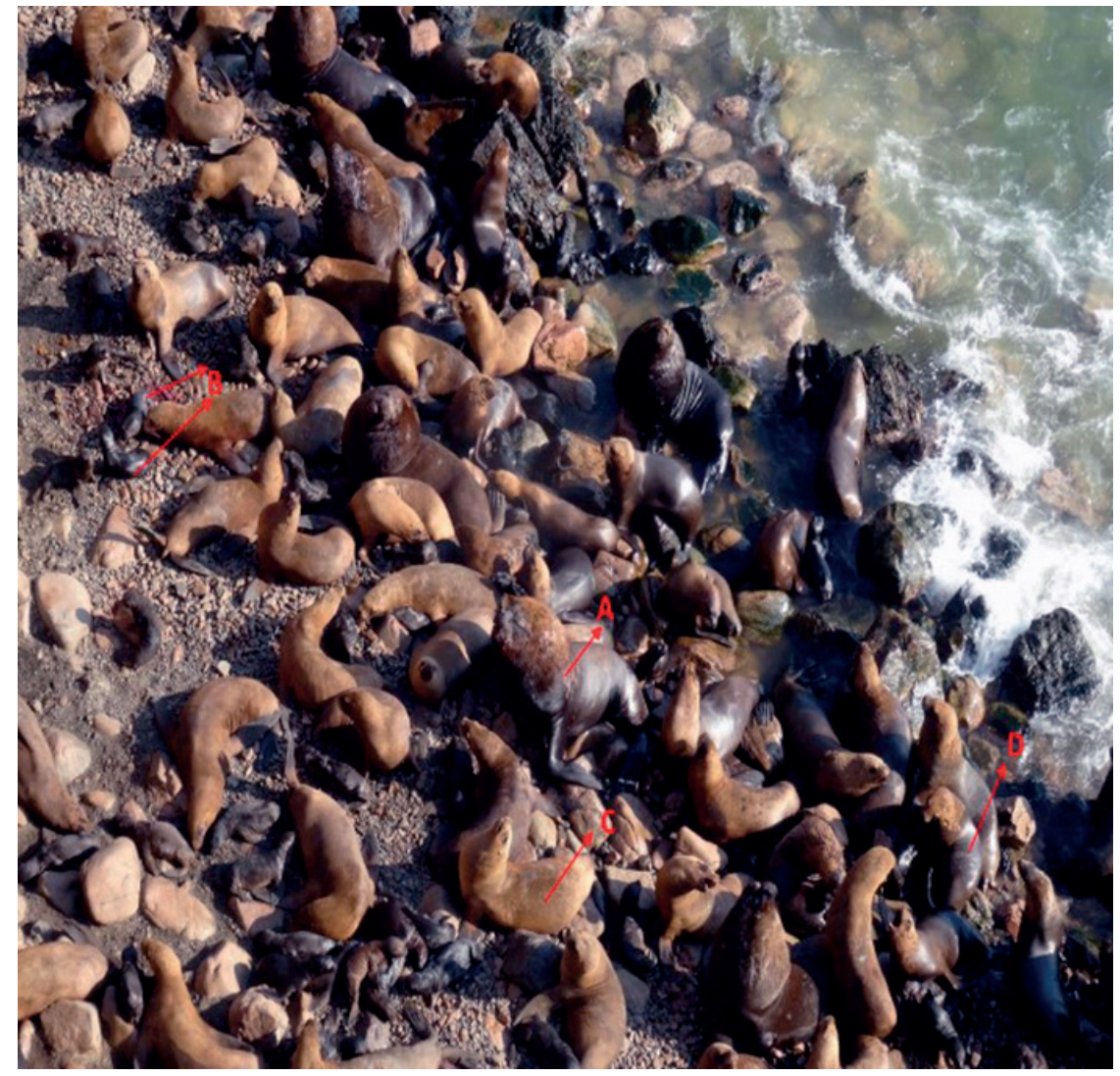

Figura 6. Categorías de lobos chuscos: A) Macho adulto, B) crías, C) Hembras, D) Juveniles. En la figura no se observa individuos de la categoría Machos subadultos (SAMs por sus siglas en inglés).

Fuente: Elaboración propia.

Crías: Animales nacidos durante la temporada reproductiva. Miden de 0.75 a $0.85 \mathrm{~m}$. Presentan pelaje oscuro lustroso durante su primera etapa. La mayor parte del tiempo, se les puede identificar fácilmente porque se encuentran jugando cerca de la madre o lactando.

Hembras: Raramente más de 1.8 metros. Cuello esbelto, perfil aguzado, sin indicio de melena. Cintura pélvica ancha, zona de la cintura escapular redondeada. Variedad de color en el pelaje (pardo o amarillento).

Juveniles: Comprende a machos y hembras de entre uno a tres años de edad, variando su longitud entre 1.20 y $1.50 \mathrm{cms}$. para los machos; y 1.12 a $1.25 \mathrm{~cm}$ para las hembras. Son más pequeños y menos robustos que las hembras, y aunque pueden confundirse por la similitud de tamaños, su identificación se hace más sencilla porque la mayoría de veces se les observa asociados a hembras de mayor tamaño.

No determinado: cuando el individuo está en el agua, o por su posición, solo se ve una parte del cuerpo y no es posible determinar su categoría de sexo o edad.

Conteo de lobos marinos en entorno de Sistemas de Información Geográfico

Se utilizó el programa ArcGis para visualizar las ortofotos generadas por el equipo RPA y su post proceso, de esta manera se realizaron los conteos de individuos de lobos marinos chuscos por categorías de sexo y edad en todas las playas de la isla, incluyendo los individuos ubicados en islotes, de manera que, de cada individuo registrado se obtuvo la información de georreferenciación.

\section{Análisis estadístico de datos}

Se utilizó el Diseño Experimental de Bloques Completamente Aleatorio (DBCA) conocido como diseño de doble vía (Montgomery, 2004), que se aplica cuando el material es heterogéneo. En este diseño las unidades experimentales son heterogéneas; las unidades homogéneas están agrupadas formando los bloques, en cada bloque se tiene un número de unidades igual al número de tratamientos; los tratamientos están 
distribuidos al azar en cada bloque, el número de repeticiones es igual al número de bloques. Bajo el siguiente modelo:

$$
y_{i j}=\mu+\tau_{i}+\beta_{j}+\varepsilon_{i j}
$$

\section{Donde:}

yij: es la observación relativa al i-ésimo tratamiento del j-ésimo bloque (variable respuesta conteos de lobos).

$\mu$ : es el promedio de cada observación

ti: es el efecto del i-ésimo tratamiento (conteo directo y conteo de ortofoto).

ßj: es el efecto del j-ésimo bloque (categorías de edad y sexo: Machos adultos, machos subadultos SAMs, crías, hembras, juveniles, no determinados).

$\varepsilon i j$ : es el error aleatorio correspondiente a la observación yij

La Hipótesis estadística es la siguiente para el tratamiento:

Ho: $u 1=u 2$

Ha: $u 1 \neq u 2$ $\alpha=0.05$ (coeficiente significación), se realizó una prueba ANOVA para contrastar la Ho.

\section{RESULTADOS}

\section{Ortofoto}

Como primer producto se obtuvo una ortofoto de aproximadamente 70 hectáreas, con una resolución de 6 centímetros por pixel (Figura 7), la cual permitió identificar la especie de estudio durante el conteo digital en entorno de sistemas de información geográfico.

El segundo producto generado fue el modelo en tres dimensiones de la Isla Chincha Sur (Figura 8), con una resolución de 10 centímetros por pixel, el cual permite comprender desde un análisis geoespacial la distribución geográfica y habitad de la especie de estudio.

\section{Conteo digital y clasificación}

La ortofoto fue trabajada empleando el programa Arcmap de ARGIS, la primera tarea antes del conteo digital fue mejorar su tonalidad aplicando los filtros de saturación y brillo (Figura 9), de esta manera se pudo contabilizar y clasificar la especie de estudio sobre el total del área mapeada (Figura 10), lo que

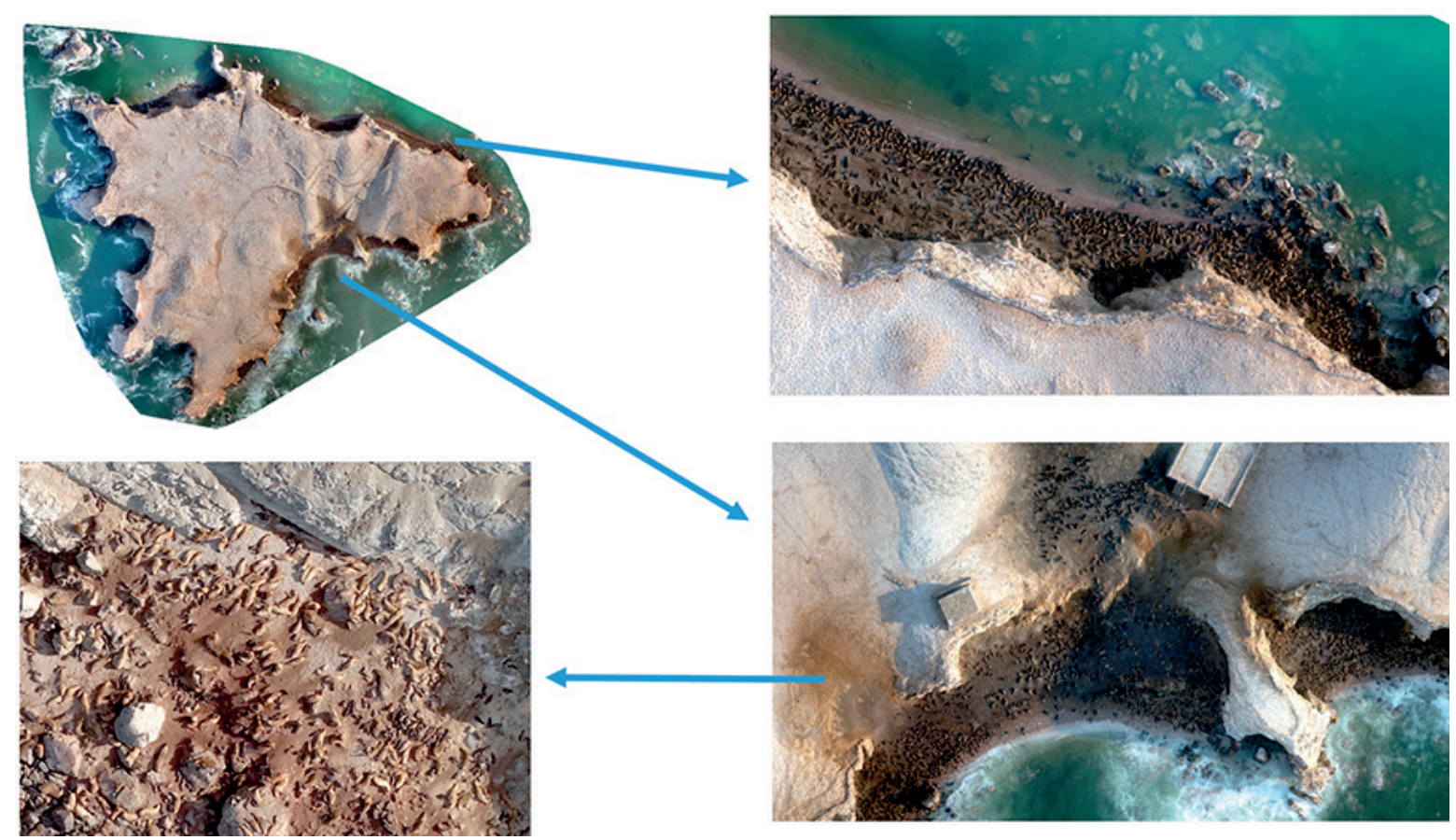

Figura 7. En la parte superior izquierda se puede apreciar la ortofoto generada sobre la Isla Chincha Sur, aproximadamente 70 hectáreas con una resolución de $6 \mathrm{~cm} /$ pix.

Fuente: Elaboración propia. 
(a)
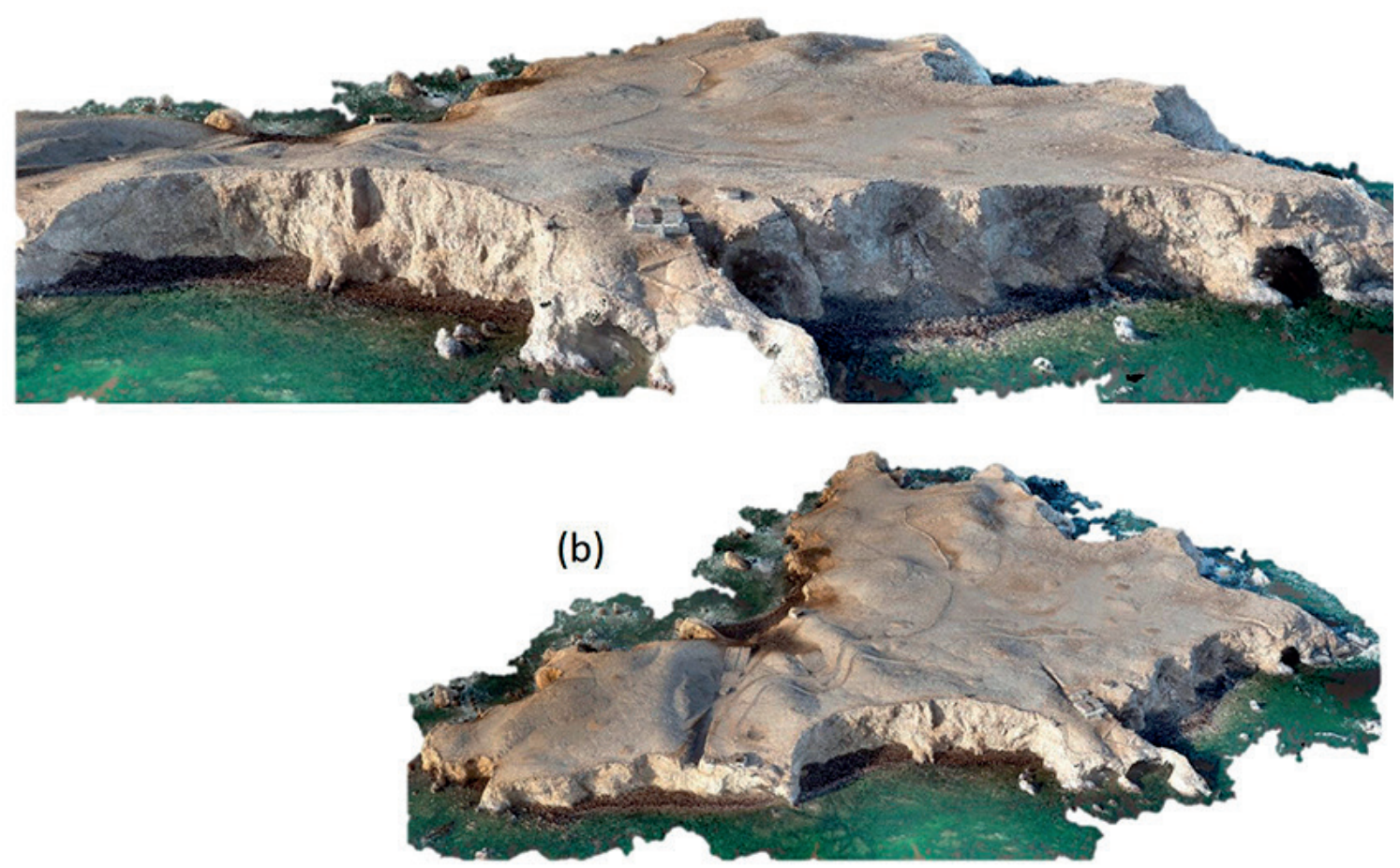

Figura 8. (a) y (b) Las vistas en tres dimensiones permiten identificar la distribución geográfica del objeto de estudio, sin perturbación del hábitat.

Fuente: Elaboración propia.

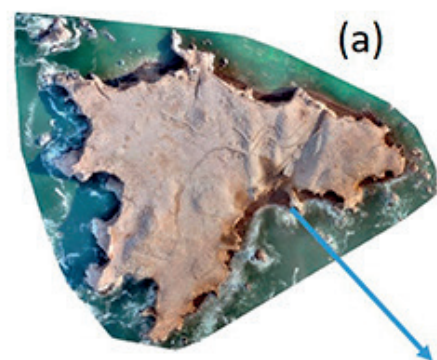

(b)

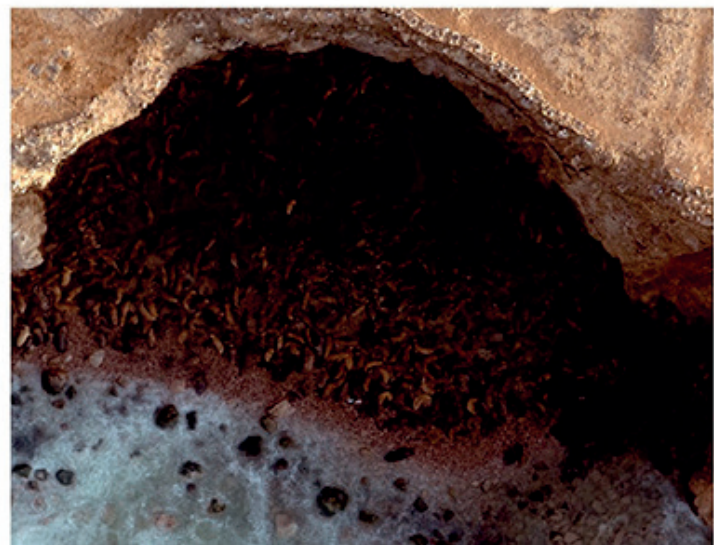

(c)

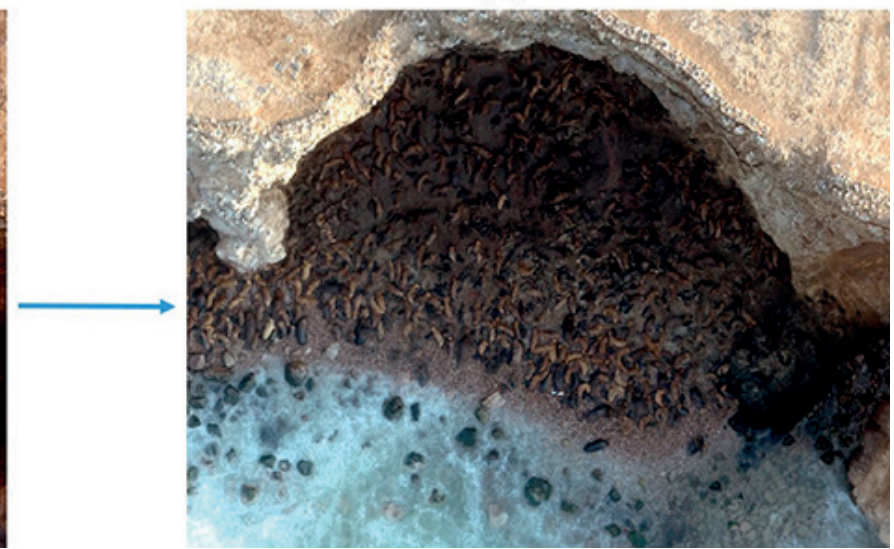

Figura 9. (a) Identificación de cueva con presencia de especie de estudio, (b) Amplificación digital donde se aprecia la especie de interés con poca claridad, (c) Zona mejorada para conteo haciendo uso de filtro de saturación y brillo.

Fuente: Elaboración propia. 


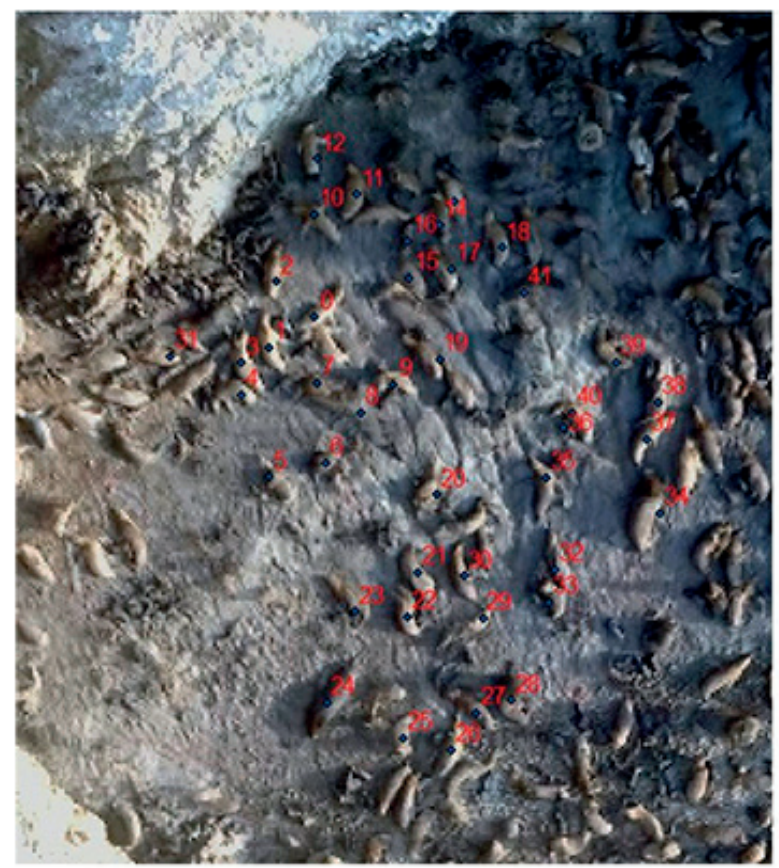

(a)
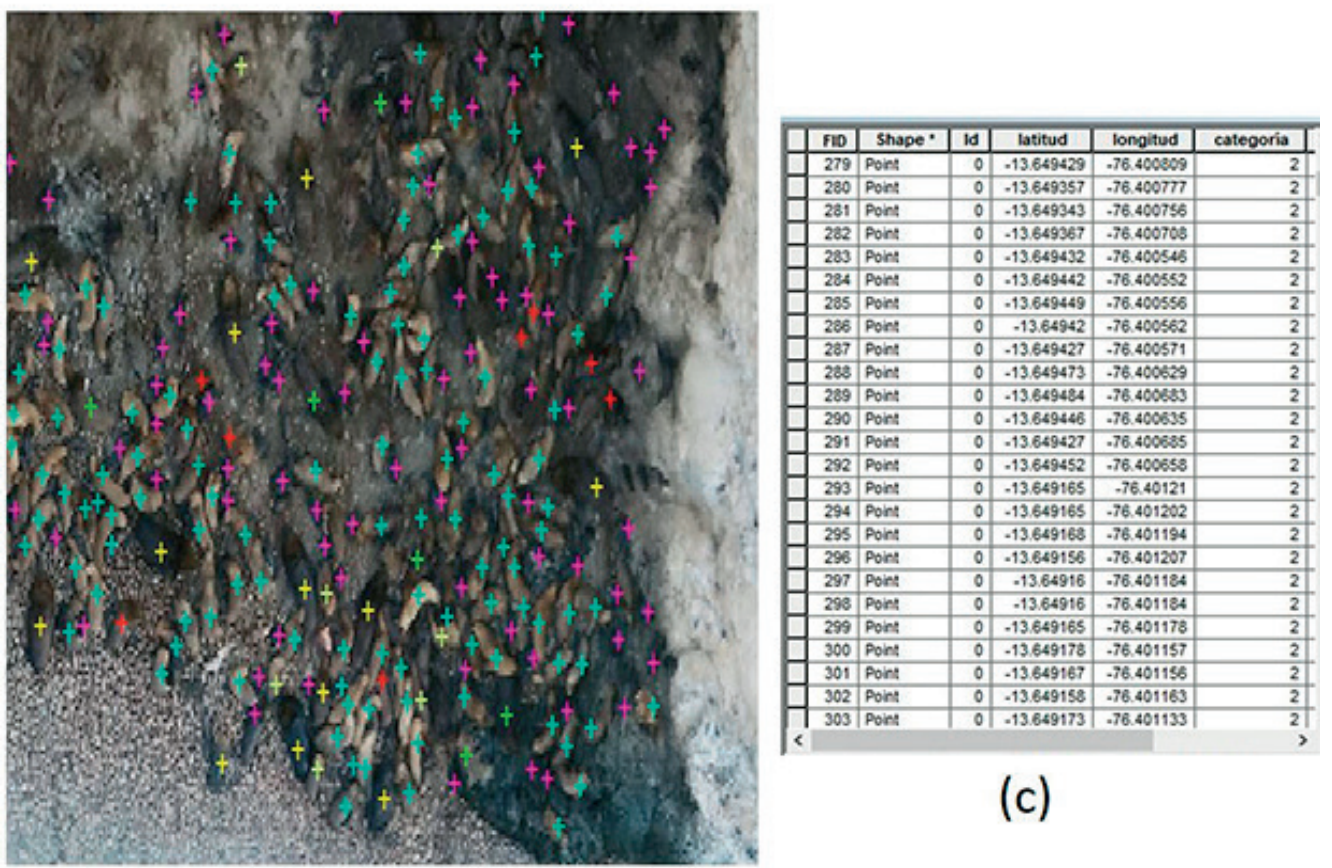

(c)

(b)

Figura 10. (a) Conteo digital, (b) Clasificación de acuerdo a su género y edad, las cruces de colores se encuentran asociados a la tabla de atributos (c), representando cada categoría tal como sigue: Cruces amarillas (categoría 1 machos), cruces rojas (categoría 2 - macho sub adulto), cruces verdes (categoría 3 - cría), cruces blancas (categoría 4 - hembra), cruces moradas (categoría 5 - juveniles), cruces celestes (categoría 6 - no determinados), (c) Tabla de atributos con referencia espacial de cada individuo identificado.

Fuente: Elaboración propia. 
además permitió contar con una tabla de atributos conteniendo metadatos de cada individuo.

El conteo digital arrojó un resultado de 2700 individuos, mientras que el conteo directo fue de 2300 individuos. Aparentemente no se aprecia mayor diferencia entre los dos métodos de conteo, sin embargo, es posible visualizar mayor variabilidad en el conteo de las ortofotos tomadas con drones (Figura $11 \mathrm{a}$ ), asimismo con el conteo digital se pudo obtener un mayor detalle del número de individuos correspondientes a las categorías crías y hembras, a diferencia del resto de categorías registradas (Figura 11 b).

\section{Análisis estadístico de datos}

De acuerdo a los resultados de la prueba ANOVA, se puede afirmar que no existen diferencias significativas entre los conteos directos y de ortofotos $\left(F_{(5,1)}=0.06 ; p=0.94\right) ;$ cabe destacar que estos datos presentan normalidad (W5 $=0.97 ; \mathrm{P}=0.98$ ) y homogeneidad de varianza $(B=0.06 ; P=0.80)$.

\section{DISCUSIÓN}

Los censos de pinnípedos se vienen realizando mediante el conteo directo de individuos en las playas de agregación y reproducción (con ayuda de binoculares, telescopios, cámaras fotográficas y contadores manuales), ya sea desde tierra o desde embarcaciones en el mar (Arias-Schreiber y Rivas, 1998; Dans et al., 2004).

Los factores causantes de la variabilidad de resultados podrían estar asociados a los cambios a los que está sujeta una población, como la hora del día, actividades de forrajeo, la altura de la marea y la época del año, entre otros factores (Eberhardt et al. (1979; Soto, Trites y Arias-Schreiber, 2012; Sepúlveda, Santos, Veas, Muñoz, Olea, Moraga, y Sielfeld, 2015; Trillmich, Meise, Kalberer, Mueller, Piedrahita, Pörschmann, Wolf y Krüger, 2016). Además, es importante recordar que en el tiempo en el que se lleva a cabo un vuelo RPA (o similar) los animales se pueden desplazar por todo el territorio introduciendo incertidumbre en su estimación (Prieto et al., 2014).

Al comparar los métodos utilizados en esta estimación, los resultados mostraron un mayor número de individuos contados a partir de las ortofotos, sin embargo, esta variación fue mínima (no significativa). Esto puede explicarse porque la evaluación se realizó en el periodo del día en el que los animales se encuentran en las playas y a que las condiciones climáticas fueron óptimas para el desarrollo de ambos métodos, con lo cual se evitaron inconvenientes (a)

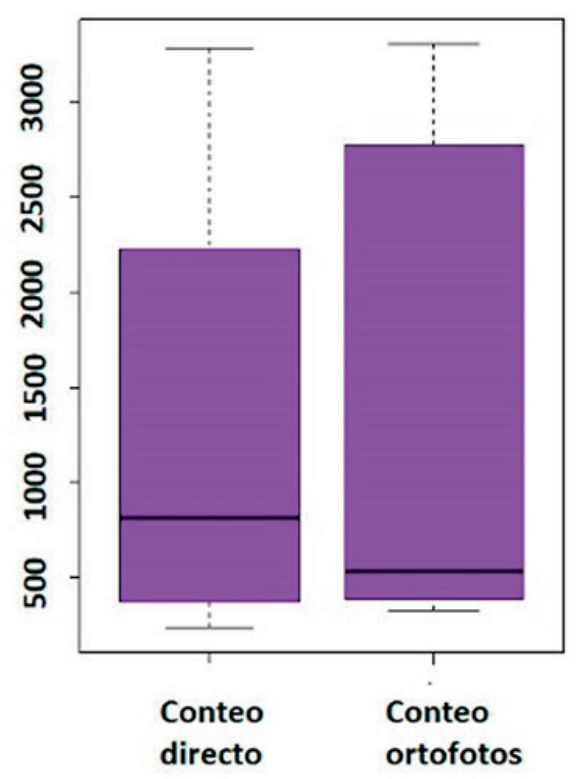

(b)

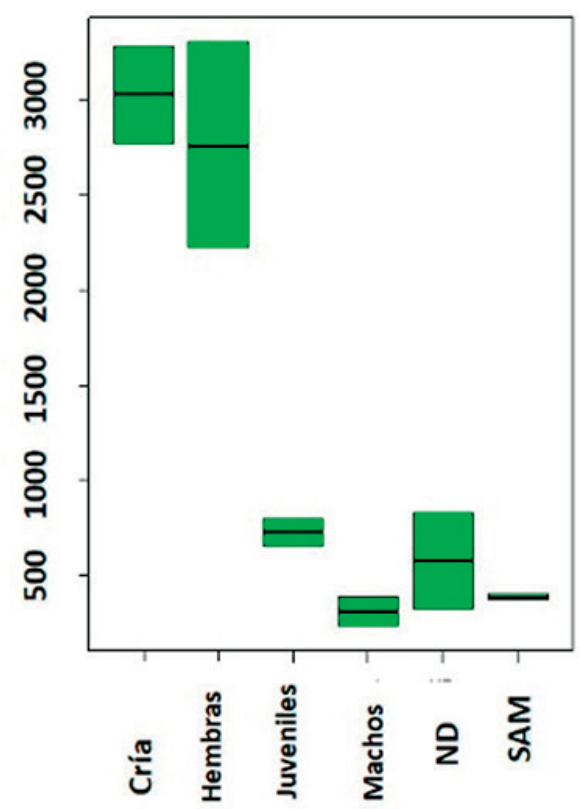

Figura 11. (a) Resultados de conteos directo y conteo de ortofotos, (b) Resultados según categoría de edad y sexo.

Fuente: Elaboración propia. 
asociados a dificultades de visibilidad y a grandes desplazamientos de animales, con los consecuentes errores en la estimación de la abundancia y categorización de individuos que implican los censos poblacionales de lobos marinos a partir de conteos directos de terreno (Sepúlveda et al. 2011).

Entre las categorías de edad y sexo registradas, las de mayor abundancia fueron las crías y hembras en ambos conteos $(71.19 \%$ del total en el conteo directo, y $77.51 \%$ del total en el conteo de ortofotos), cifras coincidentes con el periodo reproductivo en las que el número de crías es semejante al número de hembras debido al cuidado parental de la temporada, en términos de alimentación y defensa ante la presencia de depredadores (Cassini, 1999).

Entre las ventajas que podemos mencionar del empleo de RPAs en esta evaluación, están la obtención de imágenes de alta calidad en poco tiempo, la posibilidad de hacer repeticiones de conteos y categorización independientemente por diferentes personas. Adicionalmente, estas imágenes proporcionan una fuente permanente de registros fotográficos para referencia futura, las cuales no podrían ser logradas con técnicas llevadas a cabo desde tierra (Sorrell, Clarke, Holmberg y McIntosh, 2019). Por otro lado, es posible el intercambio de sensores ligeros de acuerdo a la necesidad de su empleo, lo cual lleva a las investigaciones a una óptica con puntos de vista cercanos y prácticos (Arencibia-Carballo, 2016).

Con respecto a las fotografías convencionales tomadas desde puntos de tierra o mar, estas generalmente son captadas lateralmente y no de manera completamente vertical a diferencia de las fotografías cenitales; por lo que existe la posibilidad de omisión de conteo en las fotos laterales ya que no siempre se ven todos los animales porque podrían estar ubicados detrás de los que se observan en primer plano.

Los censos de poblaciones empleando RPAS presenta la ventaja de recolectar datos sin ingresar a áreas de reproducción o áreas ecológicamente sensibles, evitando así la perturbación asociada con los monitoreos en el terreno (Hodgson et al., 2018). Durante la evaluación no se registró respuesta de los animales a la presencia del RPA (o al menos no pudo ser observada), lo cual coincide con estudios como el de Mulero-Pázmany et al. (2015) en el que atribuye esta ventaja al tamaño pequeño y al ruido reducido que estos sistemas producen, además de ser vehículos emisiones cero, punto importante cuando se trata de cuidado ambiental. Además, a medida que las plataformas RPA, los sensores y las técnicas de visión por computadora continúan desarrollándose, es probable que la precisión y la rentabilidad de los enfoques basados en RPA también sigan mejorando (Hodgson et al., 2018).

Por lo tanto, la reacción de los animales está condicionada por factores relacionados tanto con los atributos del RPA como con el modo de operación (patrones de vuelo), y por características de los animales estudiados. El patrón de vuelo del RPA tiene un efecto importante en las respuestas de la vida silvestre; así, los vuelos orientados a objetivos, como los que se realizan directamente hacia los animales y en los que generalmente se utilizan distancias cortas, principalmente para fotografía, inspecciones de nidos y recientemente para "control de animales", producen más reacciones lo que lleva a una mayor perturbación.

Por otro lado, los vuelos de cortacésped, generalmente realizados para mapeo, vigilancia y censo de vida silvestre (como en el caso del presente trabajo), realizados a alturas mayores y siguiendo trayectorias regulares tienen menos probabilidades de afectar a los animales (Mulero-Pázmány et al., 2017). Sin embargo, persiste la necesidad de realizar más estudios acerca de los impactos del uso de RPA sobre las especies de fauna a evaluar, especies acompañantes y sus hábitats.

El empleo de RPA para el conteo de pinnípedos constituye un método seguro para el personal que lleva a cabo el trabajo de campo, que permitiría el acceso a localidades que por sus características topográficas en muchos casos son difíciles de evaluar, y con un mínimo de impacto sobre las poblaciones de lobos marinos. El uso de estas herramientas ha permitido obtener importantes beneficios para la gestión ambiental, que incluyen datos de abundancia con exactitud y precisión mejorados y menores sesgos, lo cual aumenta el poder estadístico para detectar las tendencias de la población (Hodgson et al., 2018, Sorrell et al., 2019), y sus patrones de distribución, herramientas relevantes para planificaciones futuras y gestión de manejo de fauna silvestre (Mulero-Pázmány et al., 2015).

\section{CONCLUSIONES}

El empleo de RPA representa una buena alternativa y/o complemento a los conteos directos de terreno de fauna silvestre durante los monitoreos.

Entre las ventajas de la utilización del conteo de ortofotos se puede resaltar la georreferenciación de cada individuo y la posibilidad de hacer repeticiones del conteo, incluso por diferentes personas. 
El uso de esta metodología supone poca interferencia en las poblaciones de animales siempre que se lleve a cabo después de una adecuada planificación de vuelo, entrenamiento del equipo de operadores y de tomar en cuenta aspectos logísticos y operativos.

La aplicación de técnicas de procesamiento digital mejora la tonalidad de la imagen, permitiendo un conteo y categorización más precisos de los individuos en las colonias de lobos marinos.

\section{AGRADECIMIENTOS}

Esta evaluación formó parte de la actividad "Censo Nacional de Lobos Marinos Chuscos Otaria flavescens 2019" conducida por la Oficina de Investigaciones en Depredadores Superiores del Instituto del Mar del Perú. Agradecemos al Instituto Geográfico Nacional de Perú por permitir la participación de un especialista en el empleo de equipos RPA y su post procesamiento. Asimismo, a la Dra. Ana Alegre por su apoyo en el análisis estadístico, y a los guardaislas de la Isla Chincha por las facilidades para la realización del trabajo de campo.

\section{REFERENCIAS BIBLIOGRÁFICAS}

[1] Arencibia-Carballo, G. (2016). ¿Es posible el uso de drones en la investigación científica y el monitoreo ambiental? Revista Electrónica de Veterinaria, 17(9). Recuperado de http://www. veterinaria.org/revistas/redvet/n060616.html

[2] Arias-Schreiber, M. y Rivas, C. (1998). Distribución, tamaño y estructura de las poblaciones de lobos marinos (Arctocephalus australis y Otaria byronia) en el litoral peruano, en noviembre 1996 y marzo 4997. IMARPE, Informe Progresivo (73), 17-32.

[3] Berovides, V., Cañizares, M., y González, A. (2005). Métodos de Conteo de Animales y Plantas Terrestres. Manual para la capacitación del Personal técnico de las Áreas Protegidas de Cuba. Centro Nacional de Áreas Protegidas Ministerio de Ciencia, Tecnología y Medio Ambiente. La Habana, Cuba.

[4] Cassini, M (1999). The evolution of reproductive systems in pinnipeds. Behavioral Ecology, 10(5), 612-616.

[5] Contreras, F., Bartheld, J., Montecinos, M., Moreno F. y Torres, J. (2014). Cuantificación poblacional de lobo marino común (Otaria flavescens) en el litoral de la XV, I y II Regiones. Informe Final Proyecto 2012-6-FAP-
1. Recuperado de: https://www.researchgate. net/publication/278713906_Cuantificacion_ poblacional_del_lobo_marino_comun_Otaria_ flavescens_en_el_litoral_de_la_XV_I_y_II_ Regiones_2012

[6] Dans, S., Crespo, E., Pedraza, S. y Koen, M. (2004). Recovery of the South American sea lion population in northern Patagonia. Canadian Journal of Fisheries and Aquatic Sciences, 61(9): 1681-1690. doi: 10.1139/F04-105

[7] Eberhardt, L.L., Chapman, D.G. y Gilbert, J.R. (1979). A Review of Marine Mammal Census Methods. Washington: Wildlife Society.

[8] Hodgson J.C, Mott, R., Baylis, S.M., Pham, T.T., Wotherspoon, S., Kilpatrick, A.D., Raja Segaran, R., Reid, I., Terauds, A. y Koh, L.P. (2018). Drones count wildlife more accurately and precisely than humans. Methods in Ecology and Evolution 9, 1160-1167. doi: 10.1111/2041210X.12974

[9] Johnston, D. (2019). Unoccupied Aircraft Systems in Marine Science and Conservation. Annual Review of Marine Science, 11:439-463.

[10] Mandujano, S. y Rísquez-Valdepeña, A. (2019). Drones: observación de fauna y de hábitats desde el aire. Recuperado de http://www.inecol. $\mathrm{mx} /$ inecol/index.php/es/2013-06-05-10-3410/17-ciencia-hoy/563-drones-observacion-defauna-y-de-habitats-desde-el-aire

[11] Mandujano, S., Mulero-Pázmany, M. y RísquezValdepeña, A. (2017). Drones: Una nueva tecnología para el estudio y monitoreo de fauna y hábitats. Revista Agro Productividad, 10(10), 79-84.

[12] McEvoy, J., Hall, G. y McDonald, P. (2016). Evaluation of unmanned aerial vehicle shape, flight path and camera type for waterfowl surveys: disturbance effects and species recognition. PeerJ 4:e1831; doi: 10.7717/ peerj.1831.

[13] Montgomery, D. (2004). Diseño y análisis de experimentos, México, D.F. Limusa Wiley

[14] Mulero-Pázmány, M., Jenni-Eiermann, S., Strebel, N., Sattler, T., Negro, J.J. y Tablado, Z. (2017). Unmanned aircraft systems as a new source of disturbance for wildlife: A systematic review. PLoS ONE 12(6), e0178448. doi: 10.1371/journal.pone. 0178448

[15] Mulero-Pázmány, M., Barasona, J.A., Acevedo, P., Vicente, J. y Negro, J.J. (2015). Unmanned Aircraft Systems complement biologging in 
spatial ecology studies. Biology and Evolution, 5(21), 1-11. doi: 10.1002/ece3.1744

[16] Oliva, D., Sepúlveda, M., Durán, L.R., Urra, A., Sielfeld, W., Moraga, R., Pavés, G. y Muñoz, L. (2012). Cuantificación poblacional de lobos marinos en las Regiones $X-X I$ y propuesta de escenarios de manejo. Informe Final Proyecto FAP ID 4728-46-LP1. Recuperado de http:// www.oceandocs.org/handle/1834/6957

[17] Pavanato, H., Silva, K., Estima, S., Monteiro, D.y Kinas, P. (2013). Occupancy dynamics of South American Sea-Lions in Brazilian Haul-outs. Brazilian Journal of Biology, 73(5), 855-862. doi: 10.1590/S1519-698420130004000023

[18] Prieto, R., Méndez, E., Vales, J.J., Pino, I., Carpintero I.R., Granado, L., Montoya, G., Giménez de Azcárate, F., Cáceres, F. y Moreira, J.M. (2014). Aplicación de UAVs a la detección de fauna como soporte a los censos cinegéticos en ámbito piloto Finca Las Navas (Córdoba). REDIAM. Revista de teledetección: Revista de la Asociación Española de Teledetección, (41), 121-131. doi: 10.4995/raet.2014.2312

[19] Sepúlveda, M., Oliva, D., Urra, A., PérezÁlvarez, M.J., Moraga, R., Schrader, D., Inostroza, P., Melo, A., Díaz, H. y Sielfeld, W. (2011). Distribution and abundance of the South American sea lion Otaria flavescens (Carnivora: Otariidae) along the central coast off Chile. Revista Chilena de Historia Natural 84(1), 97-106

[20] Sepúlveda, M., Santos, M., Veas, R., Muñoz, L., Olea, D., Moraga, R. y Sielfeld, W. (2015). Revista de Biología Marina y Oceanografía Vol. 50, N²: 205-220. doi: 10.4067/S071819572015000300001
[21] Sorrell, K. J., Clarke, R. H., Holmberg, R. y McIntosh, R.R. (2019). Remotely piloted aircraft improve precision of capture-markresight population estimates of Australian fur seals. Ecosphere 10(8), e02812. doi: 10.1002/ ecs2.2812.

[22] Soto, K., Trites, A. y Arias-Schreiber, M. (2012). Changes in diet and maternal attendance of South American sea lions indicate changes in the marine environment and prey abundance. Marine Ecology Progress Series 312: 277-290

[23] Sweeney, K., Helker, V., Perryman, W., LeRoi, D., Fritz, L., Gelatt, T. y Angliss, R. (2016). Flying beneath the clouds at the edge of the world: using a hexacopter to supplement abundance surveys of Steller sea lions (Eumetopias jubatus) in Alaska. Journal of Unmanned Vehicle Systems 4:70-81

[24] Trillmich, F., Meise, K., Kalberer, S., Mueller, B., Piedrahita, P., Pörschmann, U., Wolf, J. y Krüger, O. (2016) On the Challenge of Interpreting Census Data: Insights from a Study of an Endangered Pinniped. PLOS ONE 11(5): e0154588.

[25] Vaz-Ferreira, R. (1982). Otaria flavescens (Shaw), South American Sea Lion. En FAO Fisheries Series 4(5) (eds), Mammals in the Seas: Small cetaceans, seals, sirenias and otters (pp. 477-495). Roma:United Nations FAO. 